\title{
Primordial black holes from Gauss-Bonnet-corrected single field inflation
}

\author{
Shinsuke Kawai $\oplus^{1, *}$ and Jinsu Kim $\odot^{2, \uparrow}$ \\ ${ }^{1}$ Department of Physics, Sungkyunkwan University, Suwon 16419, Republic of Korea \\ ${ }^{2}$ Theoretical Physics Department, CERN, 1211 Geneva 23, Switzerland
}

(Received 11 August 2021; accepted 24 September 2021; published 29 October 2021)

\begin{abstract}
Primordial black holes formed in the early Universe via gravitational collapse of over-dense regions may contribute a significant amount to the present dark matter relic density. Inflation provides a natural framework for the production mechanism of primordial black holes. For example, single field inflation models with a fine-tuned scalar potential may exhibit a period of ultra-slow roll, during which the curvature perturbation may be enhanced to become seeds of the primordial black holes formed as the corresponding scales reenter the horizon. In this work, we propose an alternative mechanism for the primordial black hole formation. We consider a model in which a scalar field is coupled to the Gauss-Bonnet term and show that primordial black holes may be seeded when a scalar potential term and the Gauss-Bonnet coupling term are nearly balanced. Large curvature perturbation in this model not only leads to the production of primordial black holes, but it also sources gravitational waves at the second order. We calculate the present density parameter of the gravitational waves and discuss the detectability of the signals by comparing them with sensitivity bounds of future gravitational wave experiments.
\end{abstract}

DOI: $10.1103 /$ PhysRevD.104.083545

\section{INTRODUCTION}

Primordial black holes [1-4] are a viable candidate for dark matter $[5,6]$ and may constitute all or a part of the dark matter relic density today. Recent studies of primordial black holes as a dark matter candidate include Refs. [7-14]. Unlike astrophysical black holes, primordial black holes may form in the early Universe through the gravitational collapse of large over-dense regions; see, e.g., Refs. [14-19] for reviews.

Cosmic inflation provides a natural framework for the production of primordial black holes. Single field inflation is capable of generating large primordial curvature perturbation in small scales compared to the scale of the cosmic microwave background [20-32]. Once the mode with large curvature perturbation reenters the horizon, gravitational collapse may occur, thereby forming primordial black holes. Single field models with a canonical kinetic term and minimal coupling to gravity, however, require a severe fine-tuning of the scalar potential to generate an inflection point near the end of inflation that produces large enough density perturbations [33]. It has also been pointed out that the standard slow-roll approximation breaks down near the inflection point [33-35]. In multifield inflation, e.g., in

\footnotetext{
*kawai@skku.edu

jinsu.kim@cern.ch
}

Published by the American Physical Society under the terms of the Creative Commons Attribution 4.0 International license. Further distribution of this work must maintain attribution to the author(s) and the published article's title, journal citation, and DOI. hybrid inflation models, generation of primordial black holes is less challenging; see Refs. [36-49]. Other models for generating large primordial curvature perturbation during inflation include the one with the modified gravity sector, and with a non-canonical form of the inflaton; see e.g., Refs. [50-59]. In Ref. [60] the production of primordial black holes is discussed in the effective field theory framework. It is also possible to produce primordial black holes during (p)reheating [61-64].

The enhancement in the curvature power spectrum not only seeds the primordial black holes but may also act as a source for the tensor perturbations at the nonlinear order, producing scalar-induced second-order gravitational waves [65-68]. Therefore, inflation models in which primordial black holes are formed via large enhancement of the curvature perturbation inevitably produce the scalar-induced second-order gravitational waves. Detection or nondetection of gravitational wave signals may thus confirm or constrain the mass ranges of the primordial black holes. For example, from the pulsar timing array experiments, a relatively large mass range of $\left[0.1 M_{\odot}, 10 M_{\odot}\right]\left(M_{\odot}\right.$ is the solar mass) is strongly constrained $[69,70]$. Recently, the NANOGrav Collaboration reported a hint for stochastic gravitational wave signals [71]. In Refs. [72-77], the connection between the NANOGrav results and the primordial black holes is investigated. The future gravitational wave experiments such as Laser Interferometer Space Antenna (LISA) [78,79], DECi-hertz Interferometer Gravitational wave Observatory (DECIGO) [80,81], and Big Bang Observer (BBO) [82-84], may probe smaller mass ranges of primordial black holes. 
Other recent studies on the primordial black holes and the scalar-induced second-order gravitational waves include Refs. [16,57-59,85-97].

In the single field inflation models for which the primordial black hole production and the secondary gravitational waves are studied, the gravity sector is usually assumed to be the Einstein gravity. The Einstein gravity however is by no means a complete theory. From the effective field theory viewpoint, for example, higher curvature terms are expected to arise [98]. One such higher curvature term is the Gauss-Bonnet term $R_{\mathrm{GB}}^{2} \equiv R^{2}-4 R_{\mu \nu} R^{\mu \nu}+R_{\mu \nu \rho \sigma} R^{\mu \nu \rho \sigma}$ which leads to a relatively well-behaved theory of higher curvature gravity. Phenomenological aspects of the Gauss-Bonnet correction have been studied by many authors, including dark energy [99-103], inflation [104-122], black holes [123,124], and gravitational-wave leptogenesis [125]. In Ref. [126], we investigated a model in which a scalar field $\varphi$ is coupled to the Gauss-Bonnet term and discussed the features of a de Sitter-like fixed point as an alternative to cosmic inflation; in the presence of the Gauss-Bonnet coupling term, there may exist a nontrivial de Sitter-like fixed point where the scalar potential term is balanced with the higher curvature GaussBonnet term. Near the nontrivial fixed point, the standard slow-roll approximation is invalid, and the ultra-slow-roll regime of inflation naturally arises. Furthermore, we pointed out that the primordial curvature power spectrum may become enhanced near the nontrivial de Sitter-like fixed point, which potentially leads to production of primordial black holes. In this paper, we investigate the production of primordial black holes and the scalar-induced second-order gravitational waves in such a setup.

The rest of the paper is organized as follows. In Sec. II, we introduce our benchmark model. We adopt the natural inflation model for the scalar potential and consider a smeared step function for the Gauss-Bonnet coupling function. We choose two benchmark parameter sets and discuss enhancements in the primordial curvature power spectrum. In Sec. III, we examine the production of primordial black holes. For the chosen benchmark sets, the produced primordial black holes are shown to constitute all or a part of the dark matter relic today, at different mass scales. The two chosen benchmark sets give rise to the scalar-induced second-order gravitational wave signals peaked at two distinct frequencies. The shape and the magnitude of the gravitational wave energy density are discussed in Sec. IV, together with the detectability of the signals in the near future. We conclude in Sec. V with some comments.

\section{BENCHMARK MODEL}

We consider the action

$S=\int d^{4} x \sqrt{-g}\left\{\frac{M_{\mathrm{P}}^{2}}{2} R-\frac{1}{2} \partial_{\mu} \varphi \partial^{\mu} \varphi-V(\varphi)-\frac{\xi(\varphi)}{16} R_{\mathrm{GB}}^{2}\right\}$, where $M_{\mathrm{P}} \equiv 1 / \sqrt{8 \pi G}=2.44 \times 10^{18} \mathrm{GeV}$ is the reduced Planck mass, and $R_{\mathrm{GB}}^{2} \equiv R^{2}-4 R_{\mu \nu} R^{\mu \nu}+R^{\mu \nu \rho \sigma} R_{\mu \nu \rho \sigma}$ is the Gauss-Bonnet term (the four-dimensional Euler density). The coupling function is chosen to be ${ }^{1}$

$$
\xi(\varphi)=\xi_{0} \tanh \left[\xi_{1}\left(\varphi-\varphi_{c}\right)\right]
$$

and for the scalar potential we assume that of the natural inflation model [129-132] (see also Refs. [133-135]),

$$
V(\varphi)=\Lambda^{4}\left(1+\cos \frac{\varphi}{f}\right)
$$

The generation of primordial black holes and the induced second-order gravitational waves in the natural inflation model (3) (without the Gauss-Bonnet correction) has been discussed, e.g., in Refs. [86,88,92,93,136], where the axion (inflaton) field is coupled to gauge fields. References [56,58] discuss primordial black holes and the gravitational waves in the natural inflation model with the modified inflaton kinetic sector.

The background equations of motion for the action (1) read

$$
\begin{gathered}
3 M_{\mathrm{P}}^{2} H^{2}=\frac{1}{2} \dot{\varphi}^{2}+V+\frac{3}{2} H^{3} \xi_{, \varphi} \dot{\varphi}, \\
\ddot{\varphi}+3 H \dot{\varphi}+V_{, \varphi}+\frac{3}{2} H^{2}\left(\dot{H}+H^{2}\right) \xi_{, \varphi}=0,
\end{gathered}
$$

where ${ }_{, \varphi} \equiv d / d \varphi$ and ${ }^{*} \equiv d / d t$ with $t$ being the cosmic time. $H \equiv \dot{a} / a$ is the Hubble parameter ( $a$ is the scale factor). The model (1) exhibits a nontrivial fixed point $\varphi_{*}$ that satisfies [126]

$$
\left.\left[V_{, \varphi}+\frac{V^{2}}{6 M_{\mathrm{P}}^{4}} \xi_{, \varphi}\right]\right|_{\varphi=\varphi_{*}}=0
$$

which follows from Eqs. (4) and (5) at stationarity. Note that $\xi_{, \varphi} \propto \operatorname{sech}^{2}\left[\xi_{1}\left(\varphi-\varphi_{c}\right)\right]$. It is natural to choose $\varphi_{c}$ to be the fixed point, i.e., $\varphi_{*}=\varphi_{c}$. The condition $\varphi_{*}=\varphi_{c}$ is realized when

\footnotetext{
${ }^{1}$ This form of the coupling is motivated in microscopic physics as follows. In calculable examples of type II and heterotic compactifications, the $R_{\mathrm{GB}}^{2}$ coupling to moduli typically occurs as one-loop gravitational threshold corrections, which are determined by the spectrum of BPS states $[127,128]$. If one of the moduli $\varphi$ traverses a wall separating two domains with different spectra, the coupling function $\xi(\varphi)$ behaves as a step function across the wall. If the domain wall has a finite thickness, $\xi(\varphi)$ is modeled by a smeared step function (2). Adding a constant parameter to Eq. (2) does not alter the dynamics since that will be a topological term.
} 


$$
\xi_{1}=\frac{6 M_{\mathrm{P}}^{4} \sin \left(\varphi_{c} / f\right)}{f \Lambda^{4} \xi_{0}\left[1+\cos \left(\varphi_{c} / f\right)\right]^{2}} .
$$

The second derivative of the Gauss-Bonnet coupling function $\xi$ with respect to the field $\varphi$ at the nontrivial fixed point $\varphi_{*}$ when $\xi_{1}$ takes Eq. (7) is given by $\xi_{, \varphi \varphi}\left(\varphi=\varphi_{*}\right)=0$. Thus, the nontrivial fixed point becomes a saddle point, as pointed out in Ref. [126]. Near the nontrivial fixed point, Eq. (5) is approximated as $\ddot{\varphi}+3 H \dot{\varphi} \approx 0$, indicating an ultra-slow-roll regime. An inflaton trajectory that passes near the nontrivial fixed point will enter an ultraslow-roll regime and experiences an enhancement in the curvature power spectrum [126].

For a given inflationary background, the curvature perturbation $\zeta$ follows the equation

$$
v_{k}^{\prime \prime}+\left(C_{\zeta}^{2} k^{2}-\frac{A_{\zeta}^{\prime \prime}}{A_{\zeta}}\right) v_{k}=0,
$$

where ' denotes the conformal time $\tau$ derivative, and the quantity $v_{k}$ is $v_{k} \equiv M_{\mathrm{P}} A_{\zeta} \zeta_{k}$ with $\zeta_{k}$ being the Fourier mode of the curvature perturbation $\zeta$. The quantities $A_{\zeta}$ and $C_{\zeta}$ are given by

$$
\begin{aligned}
A_{\zeta}^{2}= & a^{2}\left(\frac{1-\sigma_{1} / 2}{1-3 \sigma_{1} / 4}\right)^{2} \\
& \times\left(2 \epsilon_{1}-\frac{1}{2} \sigma_{1}+\frac{1}{2} \sigma_{1} \sigma_{2}-\frac{1}{2} \sigma_{1} \epsilon_{1}+\frac{3}{4} \frac{\sigma_{1}^{2}}{2-\sigma_{1}}\right), \\
C_{\zeta}^{2}= & 1-\frac{a^{2}}{A_{\zeta}^{2}}\left(\frac{\sigma_{1}}{2-3 \sigma_{1} / 2}\right)^{2} \\
& \times\left(2 \epsilon_{1}+\frac{1}{4} \sigma_{1}-\frac{1}{4} \sigma_{1} \sigma_{2}-\frac{5}{4} \sigma_{1} \epsilon_{1}\right) .
\end{aligned}
$$

For derivations, see the Appendix of Ref. [126]. Here, $\epsilon_{i}$ and $\sigma_{i}$ are defined as

$\epsilon_{1} \equiv-\frac{\dot{H}}{H^{2}}, \quad \epsilon_{i>1} \equiv \frac{\dot{\epsilon}_{i-1}}{H \epsilon_{i-1}}, \quad \sigma_{1} \equiv \frac{H \dot{\xi}}{M_{\mathrm{P}}^{2}}, \quad \sigma_{i>1} \equiv \frac{\dot{\sigma}_{i-1}}{H \sigma_{i-1}}$.

For the tensor mode, the perturbation equation is given by Ref. [126]

$$
u_{k}^{\prime \prime}+\left(C_{t}^{2} k^{2}-\frac{A_{t}^{\prime \prime}}{A_{t}}\right) u_{k}=0
$$

where $u_{k}$ is the Fourier transform of $u^{ \pm}$(the superscript \pm indicates the two polarization modes, which are omitted hereafter) which is defined via

$$
h_{i j}=\frac{\sqrt{2}}{A_{t} M_{\mathrm{P}}} \sum_{ \pm} u^{ \pm} e_{i j}^{ \pm},
$$

with $e_{i j}^{ \pm}$being the polarization tensor, and

$$
\begin{gathered}
A_{t}^{2}=a^{2}\left(1-\frac{\sigma_{1}}{2}\right), \\
C_{t}^{2}=1+\frac{a^{2} \sigma_{1}}{2 A_{t}^{2}}\left(1-\sigma_{2}-\epsilon_{1}\right) .
\end{gathered}
$$

We numerically solve the background equations of motion, Eqs. (4) and (5), and the perturbation equations Eqs. (8) and (12). For the initial conditions for the perturbations, we adopt the standard Wentzel-KramersBrillouin (WKB) solutions on the Bunch-Davies vacuum,

$$
\begin{aligned}
& \lim _{\tau \rightarrow-\infty} v_{k}(\tau)=\frac{1}{\sqrt{2 C_{\zeta} k}} e^{-i C_{\zeta} k \tau}, \\
& \lim _{\tau \rightarrow-\infty} u_{k}(\tau)=\frac{1}{\sqrt{2 C_{t} k}} e^{-i C_{t} k \tau} .
\end{aligned}
$$

The power spectra for the curvature and tensor perturbations are then obtained as

$$
\begin{gathered}
\mathcal{P}_{\zeta}=\frac{k^{3}}{2 \pi^{2}}|\zeta|^{2}=\frac{k^{3}}{2 \pi^{2}} \frac{\left|v_{k}\right|^{2}}{M_{\mathrm{P}}^{2} A_{\zeta}^{2}}, \\
\mathcal{P}_{t}=2 \times \frac{k^{3}}{2 \pi^{2}}\left|h_{k}\right|^{2}=\frac{2 k^{3}}{\pi^{2}} \frac{\left|u_{k}\right|^{2}}{M_{\mathrm{P}}^{2} A_{t}^{2}},
\end{gathered}
$$

evaluated in the superhorizon limit. The spectral index $n_{s}$ and the tensor-to-scalar ratio $r$ are then given by

$$
n_{s} \equiv 1+\frac{d \ln \mathcal{P}_{\zeta}}{d \ln k}, \quad r \equiv \frac{\mathcal{P}_{t}}{\mathcal{P}_{\zeta}},
$$

evaluated at the pivot scale $k_{*}=0.05 \mathrm{Mpc}^{-1}$.

In this work, we take the two benchmark parameter sets given in Table I. The value of $\Lambda$ is fixed from $A_{s} \approx$ $2.1 \times 10^{-9}$, where $A_{s}$ is the curvature power spectrum amplitude at the pivot scale $k_{*}=0.05 \mathrm{Mpc}^{-1}$ [137]. For a given $f$, we are then left with three free parameters, $\xi_{0}, \xi_{1}$, and $\varphi_{c}$. The parameter $\varphi_{c}$ controls the peak position of the curvature perturbation, which is related to the size of the primordial black holes and the peak frequency of the induced gravitational waves as shown in the next sections, while the parameter $\xi_{1}$ controls the width of the peak.

TABLE I. Two sets of benchmark parameters.

\begin{tabular}{cccccc}
\hline \hline & $\Lambda\left(M_{\mathrm{P}}\right)$ & $f\left(M_{\mathrm{P}}\right)$ & $\varphi_{c}\left(M_{\mathrm{P}}\right)$ & $\xi_{0}\left(10^{7}\right)$ & $\xi_{1}\left(M_{\mathrm{P}}^{-1}\right)$ \\
\hline Set 1 & 0.0065 & 7 & 13.0 & 6.044 & 15.0 \\
Set 2 & 0.0065 & 7 & 11.3 & 2.795 & 18.5 \\
\hline \hline
\end{tabular}


The parameter $\xi_{0}$, which is responsible for the magnitude of the peak of the curvature perturbation, is chosen to be close to the value determined by Eq. (7). Our numerical procedure is as follows. For a given parameter set, we solve the background equations of motion, (4) and (5), with the assumption that the inflaton starts to roll down the potential (3) near the origin; thus, inflation takes place in the range $0<\varphi<f \pi$, during which the inflaton is temporarily trapped near the $\varphi=\varphi_{c}$ point, and after that, it oscillates about and settles at $\varphi=f \pi$, where $V=0$. The end of inflation is found by using the condition $\epsilon_{1}=1$ where $\epsilon_{1}$ is the first Hubble slow-roll parameter defined as Eq. (11). Choosing the number of $e$-folds of 70 for the pivot scale, we find the horizon-crossing time of the mode. We then solve the perturbation equations, (8) and (12), for a given wave number $k$. The initial conditions, (16) and (17), are used when the mode is deep inside the horizon $k \gg a H$; concretely we require $k /(a H) \sim 10^{3}$. We evolve the perturbation equations until the mode exits the horizon and resides far outside the horizon $k \ll a H$; specifically, we require $k /(a H) \sim 10^{-3}$. We then use Eqs. (18) and (19) to compute the power spectra. For set 1 and set 2, the curvature power spectrum (18) is shown in Fig. 1. As pointed out in Ref. [126], due to the ultra-slow-roll regime, we see a large enhancement in the curvature power spectrum. Finally, we compute the spectral index $n_{s}$ and the tensor-to-scalar ratio $r$ by using Eq. (20). They are given by $\left(n_{s}, r\right)=(0.96,0.079)$ for set 1 and $(0.96,0.080)$ for set 2. At the time when the fluctuations seeding the primordial black holes exit the horizon, the energy scale is $\rho^{1 / 4} \simeq 1.46(1.58) \times 10^{16} \mathrm{GeV}$ for the benchmark parameter set 1 (2).

A large enhancement in the curvature power spectrum indicates the possibility of gravitational collapse when the corresponding mode reenters the horizon, which leads to the formation of primordial black holes. Furthermore, such

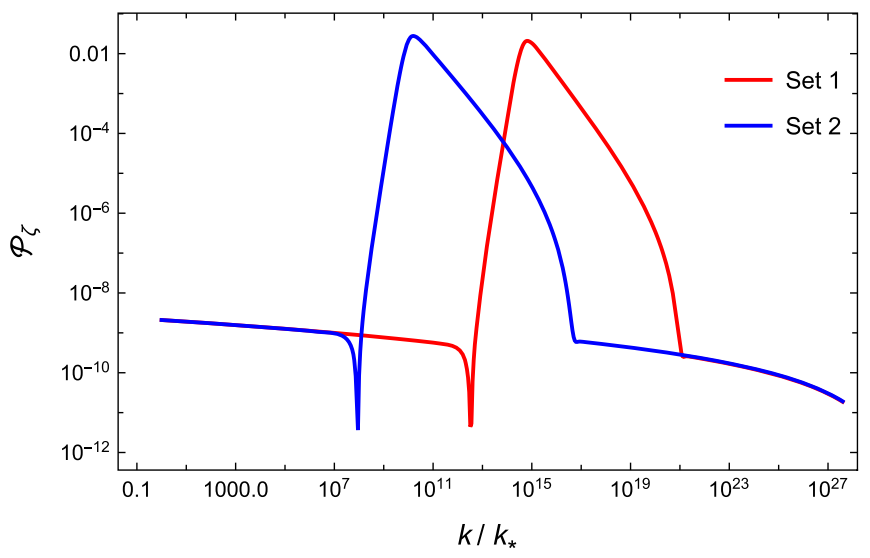

FIG. 1. The curvature power spectrum (18) is shown for our two benchmark parameter sets. The enhancement is observed as the inflaton enters the ultra-slow-roll regime near the nontrivial fixed point. Here, $k_{*}=0.05 \mathrm{Mpc}^{-1}$. a large curvature perturbation may act as a source for the tensor perturbation at the nonlinear order as the scalar mode and the tensor mode are coupled. We discuss the production of primordial black holes in the next section and the scalarinduced second-order tensor perturbation in Sec. IV.

\section{PRODUCTION OF PRIMORDIAL BLACK HOLES}

Primordial black holes may be produced due to the gravitational collapse when very large density fluctuations reenter the horizon. At its formation time $t_{\mathrm{f}}$, the mass of the produced primordial black hole is given by

$$
M=\gamma M_{H, \mathrm{f}}=4 \pi \gamma \frac{M_{\mathrm{P}}^{2}}{H_{\mathrm{f}}},
$$

where $\gamma$ is the fraction factor that accounts for how much of the horizon mass turns into the primordial black hole, and $M_{H}=4 \pi M_{\mathrm{P}}^{2} / H$ is the horizon mass. The subscript $\mathrm{f}$ in $M_{H, \mathrm{f}}$ and $H_{\mathrm{f}}$ indicates a quantity at the formation time. We use $\gamma=0.2$ as suggested by simple analytical estimations [138]. From the entropy conservation, we find that the Hubble rate at the formation time, $H_{\mathrm{f}}$, is related to the Hubble rate at present, $H_{0}$, as

$$
H_{\mathrm{f}}=H_{0} \frac{\Omega_{\mathrm{rad}, 0}^{1 / 2}}{a_{\mathrm{f}}^{2}}\left(\frac{g_{*, 0}}{g_{*, \mathrm{f}}}\right)^{\frac{1}{6}},
$$

where we have assumed that the formation occurs during the radiation-dominated era. Here, $a_{\mathrm{f}}$ is the scale factor at the formation time (the scale factor at present $a_{0}$ is set to be unity), $\Omega_{\text {rad }} \equiv \rho_{\text {rad }} / \rho_{\text {crit }}$ the radiation energy density parameter with $\rho_{\text {crit }}$ being the critical energy density of the Universe, and $g_{*}$ the effective relativistic degrees of freedom. Therefore, we find

$$
M=\gamma M_{H, 0} \Omega_{\mathrm{rad}, 0}^{1 / 2}\left(\frac{g_{*, 0}}{g_{*, \mathrm{f}}}\right)^{\frac{1}{6}}\left(\frac{k_{0}}{k_{\mathrm{f}}}\right)^{2} .
$$

The primordial black holes behave as matter. Thus, they redshift as $\rho_{\mathrm{PBH}} \propto a^{-3}$. Then, we obtain $\rho_{\mathrm{PBH}, 0}=$ $\rho_{\text {PBH,f }}\left(a_{\mathrm{f}} / a_{0}\right)^{3} \approx \gamma \beta \rho_{\text {rad,f }}\left(a_{\mathrm{f}} / a_{0}\right)^{3}$, where $\beta$ is the probability that the density fluctuation $\delta$ exceeds a threshold value $\delta_{c}$ for a given curvature power spectrum $\mathcal{P}_{\zeta}$, which is, assuming that the density fluctuation follows a Gaussian distribution, ${ }^{2}$ given by

$$
\beta(M)=\int_{\delta_{c}} d \delta \frac{1}{\sqrt{2 \pi \sigma^{2}}} \exp \left(-\frac{\delta^{2}}{2 \sigma^{2}}\right)
$$

\footnotetext{
${ }^{2}$ For the effects of non-Gaussianities on the primordial black hole formation and the scalar-induced second-order gravitational waves, see e.g., Refs. [20,139-159].
} 


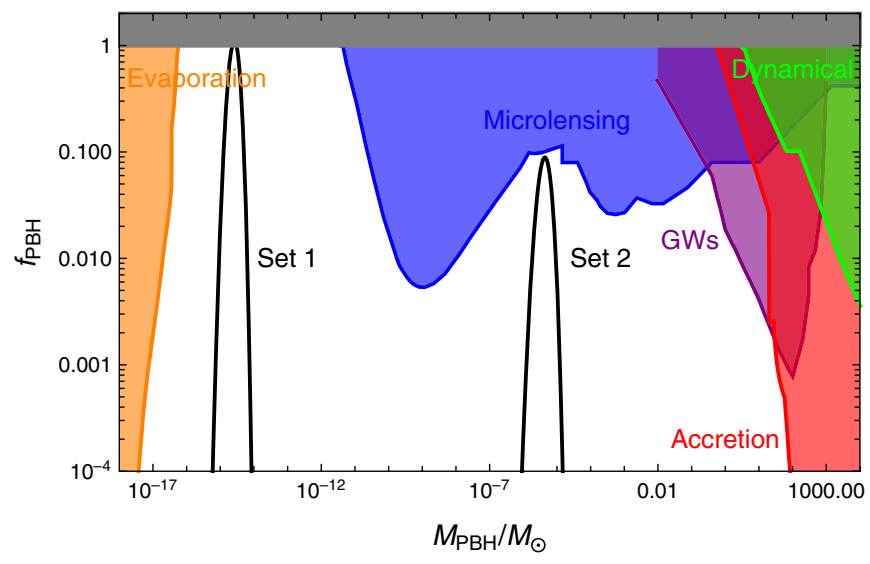

FIG. 2. The abundance of primordial black holes is shown for our two benchmark parameter sets. Here, $f_{\mathrm{PBH}} \equiv \Omega_{\mathrm{PBH}, 0} /$ $\Omega_{\mathrm{CDM}, 0}$. For the case of set 1, primordial black holes constitute all of the dark matter abundance today. For the case of set 2, only a part of the present dark matter abundance is accounted for by the primordial black holes. The data for the constraints are obtained from Refs. [18,162]. A recent analysis indicates more severe bounds from the gravitational waves [163].

with the variance $[160,161]$

$$
\sigma^{2}=\frac{16}{81} \int_{0}^{\infty} \frac{d q}{q}\left(\frac{q}{k}\right)^{4} W^{2}\left(\frac{q}{k}\right) \mathcal{P}_{\zeta}(q)
$$

We take the Gaussian window function $W(q / k)=$ $\exp \left(-(q / k)^{2} / 2\right)$ and use $\delta_{c}=1 / 3$ [138].

The total abundance of the primordial black holes is given by $\Omega_{\mathrm{PBH}, \text { tot }}=\int d \ln M \Omega_{\mathrm{PBH}}$, where $\Omega_{\mathrm{PBH}}$ is conventionally expressed in terms of the quantity $f_{\mathrm{PBH}}$ given by

$$
\begin{aligned}
f_{\mathrm{PBH}} \equiv & \frac{\Omega_{\mathrm{PBH}, 0}}{\Omega_{\mathrm{CDM}, 0}}=\gamma^{\frac{3}{2}} \beta(M) \frac{\Omega_{\mathrm{rad}, 0}^{3 / 4}}{\Omega_{\mathrm{CDM}, 0}}\left(\frac{g_{*, 0}}{g_{*, \mathrm{f}}}\right)^{\frac{1}{4}}\left(\frac{M_{H, 0}}{M}\right)^{\frac{1}{2}}, \\
\approx & \left(\frac{\beta(M)}{3.27 \times 10^{-8}}\right)\left(\frac{\gamma}{0.2}\right)^{\frac{3}{2}}\left(\frac{106.75}{g_{*, \mathrm{f}}}\right)^{\frac{1}{4}} \\
& \times\left(\frac{0.12}{\Omega_{\mathrm{CDM}, 0} h^{2}}\right)\left(\frac{M}{M_{\odot}}\right)^{-\frac{1}{2}},
\end{aligned}
$$

where $\Omega_{\mathrm{CDM}, 0}$ is the current density parameter of the cold dark matter, $M_{\odot}$ the solar mass, and $h$ the rescaled presentday Hubble rate defined by $H_{0}=100 h \mathrm{~km} / \mathrm{s} / \mathrm{Mpc}$. Here, we used the relation between the mass of the primordial black hole $M$ and a scale $k$,

$$
M(k) \approx 4.64 \times 10^{15} \gamma M_{\odot}\left(\frac{g_{*}}{106.75}\right)^{-1 / 6}\left(\frac{k}{k_{*}}\right)^{-2},
$$

where $k_{*}=0.05 \mathrm{Mpc}^{-1}$ is the pivot scale.

In Fig. 2, we present $f_{\mathrm{PBH}}$ for the two sets of parameter values, together with the current constraints $[18,162]$. For set 1 , we obtain $f_{\mathrm{PBH}}^{\text {tot }} \approx 1$, where $f_{\mathrm{PBH}}^{\text {tot }} \equiv \int d \ln M f_{\mathrm{PBH}}$.
Thus, the primordial black holes may constitute all of the dark matter abundance today. For set 2, we have $f_{\mathrm{PBH}}^{\text {tot }} \approx 0.087$.

\section{DETECTABILITY OF INDUCED GRAVITATIONAL WAVES}

The enhanced curvature perturbation may become a source for the tensor perturbation at the nonlinear order [65-68]; see also Refs. [164-168]. It is due to the fact that the scalar mode and the tensor mode couple to each other at the second order in perturbations. To study the tensor perturbation induced by the second-order scalar perturbation, we work in the conformal Newtonian gauge, ${ }^{3}$ where the metric is given by

$d s^{2}=-(1+2 \Phi) d t^{2}+a^{2}\left[(1-2 \Psi) \delta_{i j}+\frac{1}{2} h_{i j}\right] d x^{i} d x^{j}$,

with $\Phi$ and $\Psi$ being scalar perturbations and $h_{i j}$ the transverse-traceless tensor perturbation to the second order. We neglect the first-order contribution to the tensor perturbation below. Furthermore, we neglect the vector perturbation and the anisotropic stress tensor, and thus, $\Phi=\Psi$. The energy density of the gravitational waves in the subhorizon region is given by Refs. [174,175]

$$
\rho_{\mathrm{GW}}=\frac{M_{\mathrm{P}}^{2}}{16 a^{2}}\left\langle\overline{\partial_{k} h_{i j} \partial^{k} h^{i j}}\right\rangle,
$$

where the overline indicates the average over oscillations, and the angle brackets denote the expectation value. In the Fourier space, the tensor mode $h_{i j}$ can be decomposed into two polarization modes,

$h_{i j}(t, \mathbf{x})=\int \frac{d^{3} k}{(2 \pi)^{3 / 2}}\left(h_{\mathbf{k}}^{+}(t) e_{i j}^{+}(\mathbf{k})+h_{\mathbf{k}}^{\times}(t) e_{i j}^{\times}(\mathbf{k})\right) e^{i \mathbf{k} \cdot \mathbf{x}}$,

where the polarization tensors are given by

$$
\begin{aligned}
& e_{i j}^{+}(\mathbf{k})=\frac{1}{\sqrt{2}}\left(e_{i}(\mathbf{k}) e_{j}(\mathbf{k})-\bar{e}_{i}(\mathbf{k}) \bar{e}_{j}(\mathbf{k})\right), \\
& e_{i j}^{\times}(\mathbf{k})=\frac{1}{\sqrt{2}}\left(e_{i}(\mathbf{k}) \bar{e}_{j}(\mathbf{k})+\bar{e}_{i}(\mathbf{k}) e_{j}(\mathbf{k})\right),
\end{aligned}
$$

with $e_{i}(\mathbf{k})$ and $\bar{e}_{i}(\mathbf{k})$ being two orthogonal unit vectors that are perpendicular to k. Substituting Eq. (30) into Eq. (29), we obtain

\footnotetext{
${ }^{3}$ For the issue of gauge dependence, see, e.g., Refs. [169-173].
} 


$$
\rho_{G W}(t)=\int d \ln k \frac{M_{\mathrm{P}}^{2}}{8}\left(\frac{k}{a}\right)^{2} \overline{\mathcal{P}_{h}(t, k)},
$$

where $\mathcal{P}_{h}(t, k) \equiv \mathcal{P}_{h}^{+, \times}(t, k)$ is the tensor power spectrum, defined by

$$
\begin{aligned}
\left\langle h_{\mathbf{k}}^{+}(t) h_{\mathbf{q}}^{+}(t)\right\rangle & =\delta^{3}(\mathbf{k}+\mathbf{q}) \frac{2 \pi^{2}}{k^{3}} \mathcal{P}_{h}^{+}(t, k), \\
\left\langle h_{\mathbf{k}}^{\times}(t) h_{\mathbf{q}}^{\times}(t)\right\rangle & =\delta^{3}(\mathbf{k}+\mathbf{q}) \frac{2 \pi^{2}}{k^{3}} \mathcal{P}_{h}^{\times}(t, k) .
\end{aligned}
$$

Note that $\mathcal{P}_{h}^{+}(t, k)=\mathcal{P}_{h}^{\times}(t, k)$ in the absence of $C P$ violation. In the following, we omit the polarization index. It is conventional to define the energy density parameter of the gravitational waves as

$$
\Omega_{\mathrm{GW}}(t, k) \equiv \frac{\rho_{\mathrm{GW}}(t, k)}{\rho_{\text {crit }}}=\frac{1}{24}\left(\frac{k}{a H}\right)^{2} \overline{\mathcal{P}_{h}(t, k)},
$$

where $\rho_{\text {crit }}$ is the critical energy density of the Universe.

The tensor power spectrum is obtained by solving the equation of motion for the tensor perturbation which is given by the transverse-traceless component of the secondorder Einstein equation. The equation of motion is given, in the Fourier space, by Refs. $[68,87,167]$

$$
h_{\mathbf{k}}^{\prime \prime}+2 \mathcal{H} h_{\mathbf{k}}^{\prime}+k^{2} h_{\mathbf{k}}=4 S_{\mathbf{k}},
$$

where $\mathcal{H} \equiv a^{\prime} / a=a H$, and $S_{\mathbf{k}}$ is the source term,

$$
\begin{aligned}
S_{\mathbf{k}}= & \int \frac{d^{3} q}{(2 \pi)^{3 / 2}} e_{i j}(\mathbf{k}) q_{i} q_{j}\left[2 \Phi_{\mathbf{q}} \Phi_{\mathbf{k}-\mathbf{q}}\right. \\
& \left.+\frac{4}{3(1+w)}\left(\mathcal{H}^{-1} \Phi_{\mathbf{q}}^{\prime}+\Phi_{\mathbf{q}}\right)\left(\mathcal{H}^{-1} \Phi_{\mathbf{k}-\mathbf{q}}^{\prime}+\Phi_{\mathbf{k}-\mathbf{q}}\right)\right] .
\end{aligned}
$$

Here, $w$ is the equation of state parameter. The solution can be obtained by means of Green's function. We assume that the scalar-induced second-order gravitational waves are produced during the radiation-dominated era. The energy density parameter at the production time, $\Omega_{\mathrm{GW}, \mathrm{f}}(k)=$ $\Omega_{\mathrm{GW}}\left(t_{\mathrm{f}}, k\right)$, is given by Ref. [167]

$$
\begin{aligned}
\Omega_{\mathrm{GW}, \mathrm{f}}(k)= & \frac{1}{12} \int_{0}^{\infty} d v \int_{|1-v|}^{1+v} d u\left(\frac{4 v^{2}-\left(1+v^{2}-u^{2}\right)^{2}}{4 u v}\right)^{2} \\
& \times \mathcal{P}_{\zeta}(k v) \mathcal{P}_{\zeta}(k u)\left(\frac{3\left(u^{2}+v^{2}-3\right)}{4 u^{3} v^{3}}\right)^{2} \\
& \times\left[\left(-4 u v+\left(u^{2}+v^{2}-3\right) \log \left|\frac{3-(u+v)^{2}}{3-(u-v)^{2}}\right|\right)^{2}\right. \\
& \left.+\pi^{2}\left(u^{2}+v^{2}-3\right)^{2} \theta(v+u-\sqrt{3})\right] .
\end{aligned}
$$

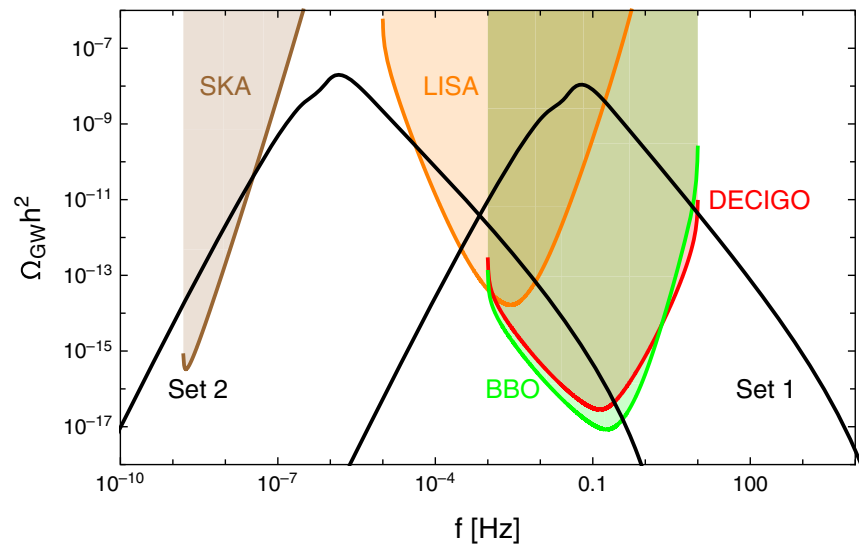

FIG. 3. The density parameter of the scalar-induced secondorder gravitational waves is shown for our two benchmark sets. The gravitational wave signal of set 1 is well within the reach of the sensitivity bound of future experiments such as LISA, DECIGO, and BBO. In the case of set 2, the signal crosses the sensitivity bound of SKA as well. The data for the sensitivity curves are obtained from Refs. [180,181].

The density parameter today is then given by Ref. [167] (see also Ref. [176]),

$$
\Omega_{\mathrm{GW}}=\Omega_{\mathrm{rad}, 0} \Omega_{\mathrm{GW}, \mathrm{f}},
$$

where $\Omega_{\text {rad }, 0} \approx 0.9 \times 10^{-4}$ is the current energy density parameter of radiation.

Utilizing Eqs. (39) and (40), together with the curvature power spectrum in Fig. 1, we present the gravitational waves density parameter in Fig. 3 for our two sets of parameter values. The gravitational wave signal of set 1 is within the reach of future experiments such as LISA [78,79], DECIGO [80,81], and BBO [82-84]. For set 2, the signal crosses the sensitivity bound of Square Kilometer Array (SKA) [177-179] in addition to that of LISA, DECIGO, and BBO.

\section{FINAL REMARKS}

We studied the production of primordial black holes and the scalar-induced second-order gravitational wave signals in a model where a scalar (inflaton) is coupled to the GaussBonnet term. The presence of the Gauss-Bonnet coupling function indicates the existence of a nontrivial de Sitter-like fixed point. Near the nontrivial fixed point, the inflaton enters an ultra-slow-roll regime. During the ultra-slow-roll inflation period, the curvature power spectrum gets enhanced. We considered two benchmark parameter sets and showed that a large enhancement occurs in the curvature power spectrum by numerically solving the equations of motion.

A mode with large enhancement of the curvature perturbation may experience gravitational collapse when reentering the horizon, thereby producing primordial black 
holes. For our two benchmark sets, we computed the present abundance of primordial black holes. One set accounts for the totality of the dark matter relic density today, while in the other case primordial black holes constitute only a portion of the present dark matter relic abundance.

A large curvature perturbation that leads to the production of primordial black holes inevitably source the scalarinduced second-order gravitational waves. The present density parameter of the gravitational waves is obtained by utilizing the approximated analytical expression together with our numerical results of the curvature power spectrum. Both of our two benchmark sets are found to be within the sensitivity bounds of future gravitational wave experiments such as LISA, DECIGO, BBO, and SKA.

While we focused on the scalar potential of the natural inflation model and assumed a smeared step function for the Gauss-Bonnet coupling function in this work, some of the features that we have found are generic. When there is a balance between a scalar potential term and a GaussBonnet coupling term, a nontrivial fixed point may exist. Near the nontrivial fixed point, the ultra-slow-roll inflation generically occurs, during which period a large enhancement of the curvature perturbation is guaranteed. We thus expect that the production of primordial black holes and the secondary gravitational wave signals are natural in higher curvature gravity theories.

\section{ACKNOWLEDGMENTS}

We acknowledge helpful communications with Kazunori Kohri and useful discussions with Valerie Domcke and Kai Schmitz. This work was supported in part by the National Research Foundation of Korea Grant-in-Aid for Scientific Research Grant No. NRF-2018R1D1A1B07051127 (S. K.).
[1] Y. B. Zel'dovich and I. D. Novikov, Sov. Astron. 10, 602 (1967), https://ui.adsabs.harvard.edu/abs/1967SvA....10. .602Z/abstract.

[2] S. Hawking, Mon. Not. R. Astron. Soc. 152, 75 (1971).

[3] B. J. Carr and S. W. Hawking, Mon. Not. R. Astron. Soc. 168, 399 (1974).

[4] A. G. Polnarev and M. Y. Khlopov, Sov. Phys. Usp. 28, 213 (1985).

[5] G. F. Chapline, Nature (London) 253, 251 (1975).

[6] P. Ivanov, P. Naselsky, and I. Novikov, Phys. Rev. D 50, 7173 (1994).

[7] D. Blais, C. Kiefer, and D. Polarski, Phys. Lett. B 535, 11 (2002).

[8] N. Afshordi, P. McDonald, and D. N. Spergel, Astrophys. J. Lett. 594, L71 (2003).

[9] P. H. Frampton, M. Kawasaki, F. Takahashi, and T. T. Yanagida, J. Cosmol. Astropart. Phys. 04 (2010) 023.

[10] K. M. Belotsky, A. D. Dmitriev, E. A. Esipova, V. A. Gani, A. V. Grobov, M. Y. Khlopov, A. A. Kirillov, S. G. Rubin, and I. V. Svadkovsky, Mod. Phys. Lett. A 29, 1440005 (2014).

[11] B. Carr, F. Kuhnel, and M. Sandstad, Phys. Rev. D 94, 083504 (2016).

[12] K. Inomata, M. Kawasaki, K. Mukaida, Y. Tada, and T. T. Yanagida, Phys. Rev. D 96, 043504 (2017).

[13] K. M. Belotsky, V. I. Dokuchaev, Y. N. Eroshenko, E. A. Esipova, M. Y. Khlopov, L. A. Khromykh, A. A. Kirillov, V. V. Nikulin, S. G. Rubin, and I. V. Svadkovsky, Eur. Phys. J. C 79, 246 (2019).

[14] B. Carr and F. Kuhnel, Annu. Rev. Nucl. Part. Sci. 70, 355 (2020).

[15] M. Y. Khlopov, Res. Astron. Astrophys. 10, 495 (2010).

[16] M. Sasaki, T. Suyama, T. Tanaka, and S. Yokoyama, Classical Quantum Gravity 35, 063001 (2018).
[17] B. Carr, K. Kohri, Y. Sendouda, and J. Yokoyama, arXiv:2002.12778.

[18] A. M. Green and B. J. Kavanagh, J. Phys. G 48, 043001 (2021).

[19] P. Villanueva-Domingo, O. Mena, and S. Palomares-Ruiz, Front. Astron. Space Sci. 8, 87 (2021).

[20] J. S. Bullock and J. R. Primack, Phys. Rev. D 55, 7423 (1997).

[21] J. Yokoyama, Phys. Rev. D 58, 083510 (1998).

[22] S. M. Leach, I. J. Grivell, and A. R. Liddle, Phys. Rev. D 62, 043516 (2000).

[23] K. Kohri, D. H. Lyth, and A. Melchiorri, J. Cosmol. Astropart. Phys. 04 (2008) 038.

[24] R. Saito, J. Yokoyama, and R. Nagata, J. Cosmol. Astropart. Phys. 06 (2008) 024.

[25] E. Bugaev and P. Klimai, Phys. Rev. D 79, 103511 (2009).

[26] L. Alabidi and K. Kohri, Phys. Rev. D 80, 063511 (2009).

[27] M. Drees and E. Erfani, J. Cosmol. Astropart. Phys. 04 (2011) 005.

[28] M. Drees and E. Erfani, J. Cosmol. Astropart. Phys. 01 (2012) 035.

[29] J. Garcia-Bellido and E. Ruiz Morales, Phys. Dark Universe 18, 47 (2017).

[30] J. M. Ezquiaga, J. Garcia-Bellido, and E. Ruiz Morales, Phys. Lett. B 776, 345 (2018).

[31] C. Germani and T. Prokopec, Phys. Dark Universe 18, 6 (2017).

[32] G. Ballesteros and M. Taoso, Phys. Rev. D 97, 023501 (2018).

[33] M. P. Hertzberg and M. Yamada, Phys. Rev. D 97, 083509 (2018).

[34] H. Motohashi and W. Hu, Phys. Rev. D 96, 063503 (2017).

[35] M. Drees and Y. Xu, Eur. Phys. J. C 81, 182 (2021).

[36] J. Silk and M. S. Turner, Phys. Rev. D 35, 419 (1987). 
[37] J. Yokoyama, Astron. Astrophys. 318, 673 (1997), http:// aa.springer.de/bibs/7318003/2300673/small.htm.

[38] L. Randall, M. Soljacic, and A. H. Guth, Nucl. Phys. B472, 377 (1996).

[39] J. Garcia-Bellido, A. D. Linde, and D. Wands, Phys. Rev. D 54, 6040 (1996).

[40] M. Kawasaki, N. Sugiyama, and T. Yanagida, Phys. Rev. D 57, 6050 (1998).

[41] M. Kawasaki, N. Kitajima, and T. T. Yanagida, Phys. Rev. D 87, 063519 (2013).

[42] K. Kohri, C. M. Lin, and T. Matsuda, Phys. Rev. D 87, 103527 (2013).

[43] S. Clesse and J. García-Bellido, Phys. Rev. D 92, 023524 (2015).

[44] K. Ando, K. Inomata, M. Kawasaki, K. Mukaida, and T. T. Yanagida, Phys. Rev. D 97, 123512 (2018).

[45] S. V. Ketov and M. Y. Khlopov, Symmetry 11, 511 (2019).

[46] G. A. Palma, S. Sypsas, and C. Zenteno, Phys. Rev. Lett. 125, 121301 (2020).

[47] M. Braglia, D. K. Hazra, F. Finelli, G. F. Smoot, L. Sriramkumar, and A. A. Starobinsky, J. Cosmol. Astropart. Phys. 08 (2020) 001.

[48] A. Ashoorioon, A. Rostami, and J. T. Firouzjaee, Phys. Rev. D 103, 123512 (2021).

[49] M. Braglia, X. Chen, and D. K. Hazra, J. Cosmol. Astropart. Phys. 03 (2021) 005.

[50] K. Kannike, L. Marzola, M. Raidal, and H. Veermäe, J. Cosmol. Astropart. Phys. 09 (2017) 020.

[51] S. Pi, Y. 1. Zhang, Q. G. Huang, and M. Sasaki, J. Cosmol. Astropart. Phys. 05 (2018) 042.

[52] D. Y. Cheong, S. M. Lee, and S. C. Park, J. Cosmol. Astropart. Phys. 01 (2021) 032.

[53] J. Lin, Q. Gao, Y. Gong, Y. Lu, C. Zhang, and F. Zhang, Phys. Rev. D 101, 103515 (2020).

[54] Z. Yi, Y. Gong, B. Wang, and Z. h. Zhu, Phys. Rev. D 103, 063535 (2021).

[55] Z. Yi, Q. Gao, Y. Gong, and Z. h. Zhu, Phys. Rev. D 103, 063534 (2021).

[56] Q. Gao, Y. Gong, and Z. Yi, Nucl. Phys. B969, 115480 (2021).

[57] P. Chen, S. Koh, and G. Tumurtushaa, arXiv:2107.08638.

[58] Z. Teimoori, K. Rezazadeh, and K. Karami, Astrophys. J. 915, 118 (2021).

[59] S. Heydari and K. Karami, arXiv:2107.10550.

[60] A. Ashoorioon, A. Rostami, and J. T. Firouzjaee, J. High Energy Phys. 07 (2021) 087.

[61] A. M. Green and K. A. Malik, Phys. Rev. D 64, 021301 (2001).

[62] B. A. Bassett and S. Tsujikawa, Phys. Rev. D 63, 123503 (2001).

[63] J. Martin, T. Papanikolaou, and V. Vennin, J. Cosmol. Astropart. Phys. 01 (2020) 024.

[64] P. Auclair and V. Vennin, J. Cosmol. Astropart. Phys. 02 (2021) 038.

[65] S. Matarrese, S. Mollerach, and M. Bruni, Phys. Rev. D 58, 043504 (1998).

[66] S. Mollerach, D. Harari, and S. Matarrese, Phys. Rev. D 69, 063002 (2004).

[67] K. N. Ananda, C. Clarkson, and D. Wands, Phys. Rev. D 75, 123518 (2007).
[68] D. Baumann, P. J. Steinhardt, K. Takahashi, and K. Ichiki, Phys. Rev. D 76, 084019 (2007).

[69] L. Lentati, S. R. Taylor, C. M. F. Mingarelli, A. Sesana, S. A. Sanidas, A. Vecchio, R. N. Caballero, K. J. Lee, R. van Haasteren, and S. Babak et al., Mon. Not. R. Astron. Soc. 453, 2576 (2015).

[70] R. M. Shannon, V. Ravi, L. T. Lentati, P. D. Lasky, G. Hobbs, M. Kerr, R. N. Manchester, W. A. Coles, Y. Levin, and M. Bailes et al., Science 349, 1522 (2015).

[71] Z. Arzoumanian et al. (NANOGrav Collaboration), Astrophys. J. Lett. 905, L34 (2020).

[72] V. Vaskonen and H. Veermäe, Phys. Rev. Lett. 126, 051303 (2021).

[73] V. De Luca, G. Franciolini, and A. Riotto, Phys. Rev. Lett. 126, 041303 (2021).

[74] K. Kohri and T. Terada, Phys. Lett. B 813, 136040 (2021).

[75] S. Sugiyama, V. Takhistov, E. Vitagliano, A. Kusenko, M. Sasaki, and M. Takada, Phys. Lett. B 814, 136097 (2021).

[76] G. Domènech and S. Pi, arXiv:2010.03976.

[77] K. Inomata, M. Kawasaki, K. Mukaida, and T. T. Yanagida, Phys. Rev. Lett. 126, 131301 (2021).

[78] P. Amaro-Seoane et al. (LISA Collaboration), arXiv: 1702.00786 .

[79] J. Baker, J. Bellovary, P. L. Bender, E. Berti, R. Caldwell, J. Camp, J. W. Conklin, N. Cornish, C. Cutler, and R. DeRosa et al. arXiv:1907.06482.

[80] N. Seto, S. Kawamura, and T. Nakamura, Phys. Rev. Lett. 87, 221103 (2001).

[81] S. Kawamura, T. Nakamura, M. Ando, N. Seto, K. Tsubono, K. Numata, R. Takahashi, S. Nagano, T. Ishikawa, and M. Musha et al. Classical Quantum Gravity 23, S125 (2006).

[82] J. Crowder and N. J. Cornish, Phys. Rev. D 72, 083005 (2005).

[83] V. Corbin and N. J. Cornish, Classical Quantum Gravity 23, 2435 (2006).

[84] G. M. Harry, P. Fritschel, D. A. Shaddock, W. Folkner, and E. S. Phinney, Classical Quantum Gravity 23, 4887 (2006); 23, 7361(E) (2006).

[85] M. Sasaki, T. Suyama, T. Tanaka, and S. Yokoyama, Phys. Rev. Lett. 117, 061101 (2016); 121, 059901(E) (2018).

[86] J. Garcia-Bellido, M. Peloso, and C. Unal, J. Cosmol. Astropart. Phys. 12 (2016) 031.

[87] K. Inomata, M. Kawasaki, K. Mukaida, Y. Tada, and T. T. Yanagida, Phys. Rev. D 95, 123510 (2017).

[88] V. Domcke, F. Muia, M. Pieroni, and L. T. Witkowski, J. Cosmol. Astropart. Phys. 07 (2017) 048.

[89] J. Garcia-Bellido and S. Nesseris, Phys. Dark Universe 18, 123 (2017).

[90] S. Bhattacharya, S. Mohanty, and P. Parashari, Phys. Rev. D 102, 043522 (2020).

[91] C. Fu, P. Wu, and H. Yu, Phys. Rev. D 101, 023529 (2020).

[92] J. P. B. Almeida, N. Bernal, D. Bettoni, and J. Rubio, J. Cosmol. Astropart. Phys. 11 (2020) 009.

[93] O. Özsoy and Z. Lalak, J. Cosmol. Astropart. Phys. 01 (2021) 040.

[94] T. Papanikolaou, V. Vennin, and D. Langlois, J. Cosmol. Astropart. Phys. 03 (2021) 053.

[95] G. Domènech, C. Lin, and M. Sasaki, J. Cosmol. Astropart. Phys. 04 (2021) 062. 
[96] C. Yuan and Q. G. Huang, arXiv:2103.04739.

[97] G. Franciolini, V. Baibhav, V. De Luca, K. K. Y. Ng, K. W. K. Wong, E. Berti, P. Pani, A. Riotto, and S. Vitale, arXiv:2105.03349.

[98] S. Weinberg, Phys. Rev. D 77, 123541 (2008).

[99] S. Nojiri, S. D. Odintsov, and M. Sasaki, Phys. Rev. D 71, 123509 (2005).

[100] T. Koivisto and D. F. Mota, Phys. Lett. B 644, 104 (2007).

[101] S. Nojiri, S. D. Odintsov, and P. V. Tretyakov, Phys. Lett. B 651, 224 (2007).

[102] L. Amendola, C. Charmousis, and S. C. Davis, J. Cosmol. Astropart. Phys. 10 (2007) 004.

[103] L. N. Granda and D. F. Jimenez, Phys. Rev. D 90, 123512 (2014).

[104] S. Kawai, M. a. Sakagami, and J. Soda, Phys. Lett. B 437, 284 (1998).

[105] S. Kawai and J. Soda, Phys. Lett. B 460, 41 (1999).

[106] M. Satoh, S. Kanno, and J. Soda, Phys. Rev. D 77, 023526 (2008).

[107] M. Satoh and J. Soda, J. Cosmol. Astropart. Phys. 09 (2008) 019.

[108] Z. K. Guo and D. J. Schwarz, Phys. Rev. D 80, 063523 (2009).

[109] Z. K. Guo and D. J. Schwarz, Phys. Rev. D 81, 123520 (2010).

[110] M. Satoh, J. Cosmol. Astropart. Phys. 11 (2010) 024.

[111] S. Koh, B. H. Lee, W. Lee, and G. Tumurtushaa, Phys. Rev. D 90, 063527 (2014).

[112] Z. Yi, Y. Gong, and M. Sabir, Phys. Rev. D 98, 083521 (2018).

[113] S. Nojiri, S. D. Odintsov, V. K. Oikonomou, N. Chatzarakis, and T. Paul, Eur. Phys. J. C 79, 565 (2019).

[114] S. D. Odintsov and V. K. Oikonomou, Phys. Lett. B 797, 134874 (2019).

[115] S. D. Odintsov, V. K. Oikonomou, and F. P. Fronimos, Nucl. Phys. B958, 115135 (2020).

[116] E. O. Pozdeeva, Eur. Phys. J. C 80, 612 (2020).

[117] V. K. Oikonomou and F. P. Fronimos, Classical Quantum Gravity 38, 035013 (2021).

[118] E. O. Pozdeeva, M. R. Gangopadhyay, M. Sami, A. V. Toporensky, and S. Y. Vernov, Phys. Rev. D 102, 043525 (2020).

[119] V. K. Oikonomou and F. P. Fronimos, Europhys. Lett. 131, 30001 (2020).

[120] S. D. Odintsov, V. K. Oikonomou, and F. P. Fronimos, Ann. Phys. (Amsterdam) 420, 168250 (2020).

[121] S. D. Odintsov, V. K. Oikonomou, F. P. Fronimos, and S. A. Venikoudis, Phys. Dark Universe 30, 100718 (2020).

[122] V. K. Oikonomou and F. P. Fronimos, Eur. Phys. J. Plus 135, 917 (2020).

[123] G. Antoniou, A. Bakopoulos, and P. Kanti, Phys. Rev. Lett. 120, 131102 (2018).

[124] B. H. Lee, W. Lee, and D. Ro, Phys. Rev. D 99, 024002 (2019).

[125] S. Kawai and J. Kim, Phys. Lett. B 789, 145 (2019).

[126] S. Kawai and J. Kim, Phys. Rev. D 104, 043525 (2021).

[127] I. Antoniadis, E. Gava, and K. S. Narain, Phys. Lett. B 283, 209 (1992).

[128] J. A. Harvey and G. W. Moore, Nucl. Phys. B463, 315 (1996).
[129] K. Freese, J. A. Frieman, and A. V. Olinto, Phys. Rev. Lett. 65, 3233 (1990).

[130] F. C. Adams, J. R. Bond, K. Freese, J. A. Frieman, and A. V. Olinto, Phys. Rev. D 47, 426 (1993).

[131] C. Savage, K. Freese, and W. H. Kinney, Phys. Rev. D 74, 123511 (2006).

[132] K. Freese and W. H. Kinney, J. Cosmol. Astropart. Phys. 03 (2015) 044.

[133] J. E. Kim, H. P. Nilles, and M. Peloso, J. Cosmol. Astropart. Phys. 01 (2005) 005.

[134] S. Dimopoulos, S. Kachru, J. McGreevy, and J. G. Wacker, J. Cosmol. Astropart. Phys. 08 (2008) 003.

[135] T. W. Grimm, Phys. Rev. D 77, 126007 (2008).

[136] E. Bugaev and P. Klimai, Phys. Rev. D 90, 103501 (2014).

[137] Y. Akrami et al. (Planck Collaboration), Astron. Astrophys. 641, A10 (2020).

[138] B. J. Carr, Astrophys. J. 201, 1 (1975).

[139] P. Ivanov, Phys. Rev. D 57, 7145 (1998).

[140] D. H. Lyth, J. Cosmol. Astropart. Phys. 05 (2012) 022.

[141] C. T. Byrnes, E. J. Copeland, and A. M. Green, Phys. Rev. D 86, 043512 (2012).

[142] E. V. Bugaev and P. A. Klimai, Int. J. Mod. Phys. D 22, 1350034 (2013).

[143] S. Young and C. T. Byrnes, J. Cosmol. Astropart. Phys. 08 (2013) 052.

[144] T. Nakama, J. Silk, and M. Kamionkowski, Phys. Rev. D 95, 043511 (2017).

[145] J. Garcia-Bellido, M. Peloso, and C. Unal, J. Cosmol. Astropart. Phys. 09 (2017) 013.

[146] G. Franciolini, A. Kehagias, S. Matarrese, and A. Riotto, J. Cosmol. Astropart. Phys. 03 (2018) 016.

[147] R. g. Cai, S. Pi, and M. Sasaki, Phys. Rev. Lett. 122, 201101 (2019).

[148] V. Atal and C. Germani, Phys. Dark Universe 24, 100275 (2019).

[149] C. Unal, Phys. Rev. D 99, 041301 (2019).

[150] S. Passaglia, W. Hu, and H. Motohashi, Phys. Rev. D 99, 043536 (2019).

[151] V. Atal, J. Garriga, and A. Marcos-Caballero, J. Cosmol. Astropart. Phys. 09 (2019) 073.

[152] G. Panagopoulos and E. Silverstein, arXiv:1906.02827.

[153] C. M. Yoo, J. O. Gong, and S. Yokoyama, J. Cosmol. Astropart. Phys. 09 (2019) 033.

[154] A. Kehagias, I. Musco, and A. Riotto, J. Cosmol. Astropart. Phys. 12 (2019) 029.

[155] J. M. Ezquiaga, J. García-Bellido, and V. Vennin, J. Cosmol. Astropart. Phys. 03 (2020) 029.

[156] C. Yuan and Q. G. Huang, arXiv:2007.10686.

[157] H. V. Ragavendra, P. Saha, L. Sriramkumar, and J. Silk, Phys. Rev. D 103, 083510 (2021).

[158] P. Adshead, K. D. Lozanov, and Z. J. Weiner, arXiv: 2105.01659.

[159] V. Atal and G. Domènech, J. Cosmol. Astropart. Phys. 06 (2021) 001.

[160] A. S. Josan, A. M. Green, and K. A. Malik, Phys. Rev. D 79, 103520 (2009).

[161] S. Young, C. T. Byrnes, and M. Sasaki, J. Cosmol. Astropart. Phys. 07 (2014) 045.

[162] B. J. Kavanagh, Zenodo, https://doi.org/10.5281/zenodo.3538999. 
[163] G. Hütsi, M. Raidal, V. Vaskonen, and H. Veermäe, J. Cosmol. Astropart. Phys. 03 (2021) 068.

[164] H. Noh and J. c. Hwang, Phys. Rev. D 69, 104011 (2004).

[165] J. c. Hwang and H. Noh, Phys. Rev. D 76, 103527 (2007).

[166] J. O. Gong, arXiv:1909.12708.

[167] K. Kohri and T. Terada, Phys. Rev. D 97, 123532 (2018).

[168] G. Domènech, Int. J. Mod. Phys. D 29, 2050028 (2020).

[169] J. C. Hwang, D. Jeong, and H. Noh, Astrophys. J. 842, 46 (2017).

[170] V. De Luca, G. Franciolini, A. Kehagias, and A. Riotto, J. Cosmol. Astropart. Phys. 03 (2020) 014.

[171] K. Inomata and T. Terada, Phys. Rev. D 101, 023523 (2020).

[172] C. Yuan, Z. C. Chen, and Q. G. Huang, Phys. Rev. D 101, 063018 (2020).

[173] G. Domènech and M. Sasaki, Phys. Rev. D 103, 063531 (2021).

[174] M. Maggiore, Phys. Rep. 331, 283 (2000).
[175] M. Maggiore, in Gravitational Waves. Vol. 1: Theory and Experiments (Oxford University Press, New York, 2007).

[176] K. Ando, K. Inomata, and M. Kawasaki, Phys. Rev. D 97, 103528 (2018).

[177] C. L. Carilli and S. Rawlings, New Astron. Rev. 48, 979 (2004).

[178] G. Janssen, G. Hobbs, M. McLaughlin, C. Bassa, A. T. Deller, M. Kramer, K. Lee, C. Mingarelli, P. Rosado, S. Sanidas et al., Proc. Sci. AASKA14 (2015) 037 [arXiv:1501.00127].

[179] A. Weltman, P. Bull, S. Camera, K. Kelley, H. Padmanabhan, J. Pritchard, A. Raccanelli, S. Riemer-Sørensen, L. Shao, S. Andrianomena et al., Pub. Astron. Soc. Aust. 37, e002 (2020).

[180] K. Schmitz, J. High Energy Phys. 01 (2021) 097.

[181] K. Schmitz, Zenodo, https://doi.org/10.5281/zenodo .3689582 . 